\title{
Title
}

\section{Chemical Bonding Origin of Glass Formation in Metal-Organic Frameworks}

Sounak Sarkar, ${ }^{1}$ Thomas Bjørn Egede Grønbech, ${ }^{1}$ Aref Mamakhel, ${ }^{1}$ Martin Bondesgaard, ${ }^{1}$ Kunihisa Sugimoto, ${ }^{2}$ Eiji Nishibori, ${ }^{3}$ Bo Brummerstedt Iversen*1

* Corresponding author: bo@chem.au.dk

\section{Abstract}

Glass-forming metal organic frameworks (MOFs) have novel applications, but the origin of their peculiar melting behavior is unclear. Here, we report synchrotron X-ray diffraction electron densities of two zeolitic imidazolate frameworks (ZIFs), the glass-forming Zn-ZIF-zni and the isostructural thermally decomposing Co-ZIF-zni. Electron density analysis shows that the $\mathrm{Zn}-\mathrm{N}$ bonds are more ionic than $\mathrm{Co}-\mathrm{N}$ bond, which have distinct covalent features. Variable temperature Raman spectra reveal onset of significant imidazolate bond weakening in Co-ZIF-zni above $673 \mathrm{~K}$. Melting can be controlled by tuning the metal-ligand and imidazole bonding strength as shown from nine solid solution structures of $\mathrm{Co}_{\mathrm{x}} \mathrm{Zn}_{1-\mathrm{x}}-\mathrm{ZIF}-\mathrm{zni}(\mathrm{x}=0.3$ to 0.003 ) were synthesized, and a mere $4 \%$ Co-doping into Zn-ZIF-zni results in thermal decomposition instead of melting. These findings advocate significant chemical space for designing new MOF glasses either by engineering the chemical bonding with ligand functionalization or by avoiding defects at the metal site in the crystal lattice.

\section{Main text}

Metal organic framework (MOF) materials are usually preferred to be crystalline for potential applications to facilitate control over pore size or ligand functionalization ${ }^{1,2}$. However, amorphous MOFs ${ }^{3}$ are $^{2}$ increasingly targeted in application e.g. for drug delivery, ${ }^{4}$ packing and delivery of enzymes, ${ }^{5}$ or hydro- 
carbon adsorption ${ }^{6}$ due to abundance of active sites, lack of grain boundaries, isotropic mechanical response and better flexibility for shape-selective adsorptions. Pioneering work on amorphous MOFs has especially concerned the MOF family of zeolitic imidazolate frameworks (ZIFs) ${ }^{7,8}$. The network topology of ZIFs consists of tetrahedral metal centres (e.g. Zn(II), Co(II)) connected by imidazolate linkers, which replicates the building block of corner sharing $\mathrm{SiO}_{4}$ tetrahedra in zeolites, Fig. 1. Contrary to the majority of MOFs that show thermal decomposition upon heating, several members of the ZIF family melt above $400{ }^{\circ} \mathrm{C},{ }^{9}$ and they form glasses when thermally quenched from the molten state. Glass-forming ZIFs have opened up new avenues of fundamental and applied research ${ }^{10}$. Pure ZIF glasses as well as fabricated glass membranes with intrinsic permanent porosity are used for gas mixture separations $\left(\mathrm{H}_{2} / \mathrm{CH}_{4}, \mathrm{CO}_{2} / \mathrm{N}_{2} \text {, and } \mathrm{CO}_{2} / \mathrm{CH}_{4} \text {, propane, propylene, and n-butane }\right)^{11,12}$ and $\mathrm{CO}_{2}$ adsorption ${ }^{13}$. ZIF glasses are also being explored as candidates for optical luminescence materials, ${ }^{14}$ non-linear optical materials, ${ }^{15}$ or quasi-solid-state-electrolytes ${ }^{16}$. Notably, ZIF glasses offer better processability for bulk application than crystalline powders ${ }^{17}$.

The archetypical ZIF-4 material containing $\mathrm{Zn}^{2+}$ ions was the first glass-forming $\mathrm{MOF}^{18}$. Coudert et al. used first-principle molecular dynamics simulation and a combination of neutron and X-ray pair distribution function analysis to demonstrate that melting of Zn-ZIF-4 involves rapid fluctuations between three-coordinated and four-coordinated $\mathrm{Zn}$-imidazolate units at picosecond time scale ${ }^{19}$. Interestingly, the isostructural Co-ZIF-4 material decomposes without melting under heat treatment. Zn-ZIF-4 and Co-ZIF-4 have similar thermal signatures when heated in argon atmosphere with solvent loss from the lattice around $220{ }^{\circ} \mathrm{C}$, followed by amorphization above $300^{\circ} \mathrm{C}$, and eventual recrystallization at about $370-400{ }^{\circ} \mathrm{C}$ to densest ZIF polymorph, the so called ZIF-zni ${ }^{20}$. Melting of Zn-ZIF-zni is observed at $590{ }^{\circ} \mathrm{C}$ followed by decomposition above $600{ }^{\circ} \mathrm{C}$ (Fig. S1a). In contrast, Co-ZIF-zni decomposes at $530{ }^{\circ} \mathrm{C}$ (Fig. S1b). It is puzzling why these two isostructural ZIFs, Zn-ZIF-zni and Co-ZIF-zni, behave 
fundamentally different upon heating. They differ only in the electronic configuration of their transition metal center with closed shell $\mathrm{d}^{10}$ for $\mathrm{Zn}(\mathrm{II})$ and open shell $\mathrm{d}^{7}$ for Co(II). This suggests that the thermal stability of individual MOFs and hence their glass-forming ability is correlated to the nature of the metalligand bonding.

Various empirical design strategies based on mixed-ligand approach have been pursued to lower the melting point temperature of ZIF-62 (and derivatives), whose structures are isomorphous to ZIF-4. ${ }^{12}$, ${ }^{21,22}$. However, to date, the physicochemical origin of the melting behaviors remains poorly understood. When addressing the correlation between the melting/decomposition process and the metal-ligand bonding in Zn-ZIF/Co-ZIF, two questions appear important: i) How does the nature of Zn-ligand bond compare to the Co-ligand bond?, and ii) What is the impact of this difference on the structural dynamics and bond strength (stability) of imidazolate linkers in different ZIFs. Here we address the questions based on analysis of electron density (ED) distributions obtained from modelling of very accurate $25 \mathrm{~K}$ synchrotron X-ray diffraction data measured to a high resolution of $\sin \theta / \lambda=1.35 \AA^{-1}$ and $1.25 \AA^{-1}$ for Zn-ZIFzni and Co-ZIF-zni respectively. ED analysis provides detailed information on chemical bonding and it has helped to form understanding of structure-property relationships in material research ${ }^{23}$. However, ED studies of MOF materials remain a severe challenge both for experimental (X-ray diffraction) and computational (ab initio) methods. The possible presence of disordered solvents in the pores, framework disorder or large atomic displacements in the flexible linkers hinders accurate modelling of X-ray structure factors, while large open shell extended molecular systems with magnetic ordering are computationally costly and suffer convergence issues. In a few cases, use of intense high-energy synchrotron X-ray radiation in combination with area detector technology has resulted in experimental ED studies of $\mathrm{MOFs}^{24,25,26}$. 
We have determined accurate EDs of Zn-ZIF-zni and Co-ZIF-zni based on multipole modelling ${ }^{27}$ of $25 \mathrm{~K}$ single crystal synchrotron X-ray diffraction data supplemented with periodic DFT calculations. ZIF-zni, the highest density polymorph was selected for this study because its crystal structure is devoid of solvents, thus avoiding potential structural disorder. Similar to ZIF-4, other ZIFs such as ZIF-1, ZIF3, ZIF-zeg, and ZIF-nog, also undergo thermal amorphization and recrystallization to ZIF-zni prior to melting/decomposition ${ }^{20,28}$. To complement the X-ray ED data we have collected multi-temperature (25$400 \mathrm{~K})$ single crystal X-ray diffraction data for structural analysis as well as multi-temperature (300-773 K) Raman spectroscopy data to investigate the structural changes in the ZIF-zni frameworks during heating. Finally, the thermal behavior of a series of nine solid solutions of $\mathrm{Co}_{\mathrm{x}} \mathrm{Zn}_{1-\mathrm{x}}-\mathrm{ZIF}-\mathrm{zni}$ (x ranging from 0.3 to 0.003 ) was analyzed by simultaneous thermogravimetric analysis and differential scanning calorimetry (TGA and DSC) to further probe the role of metal-ligand bonding in regulating the melting/decomposition process in ZIFs.

The dense Zn and Co ZIF-zni MOFs (void volume is only $4 \%$ of the unit cells) are isostructural and they belong to the tetragonal $I 4_{1} c d$ space group. In the asymmetric unit of both crystal structures, two symmetry independent metal centres form a distorted tetrahedral geometry with four imidazolate rings in a 1:2 stoichiometry (Fig. 1 and Fig. S7). The $\mathrm{N}$ atoms of the imidazolate ligands are coordinated to the respective metal centres. The metal-nitrogen $(\mathrm{M}-\mathrm{N})$ bond lengths (mean value $\sim 1.985 \AA$, Table $\mathrm{S} 2$ ) and $\angle \mathrm{N}-\mathrm{M}-\mathrm{N}$ bonds angles (mean value $\sim 109.6^{\circ}$, Table $\mathrm{S} 3$ ) in both $25 \mathrm{~K}$ crystal structures are very similar, but differences in the electron configurations of the metal ions may affect the nature and strength of chemical bonding between the metals and the ligands.

Details of the multipole modelling of the X-ray structure factors to obtain the experimental EDs are provided in the method section and supporting information. The d-orbital populations of the Co atoms 
derived from experimental ED fits well with their distorted tetrahedral geometry showing a preferential occupancy in $\mathrm{d}_{\mathrm{xy}}$ orbitals compared to $\mathrm{d}_{\mathrm{yz}}$ and $\mathrm{d}_{\mathrm{xz}}$ (Table $\mathrm{S} 4$ ). The experimental static deformation densities reveal details about the metal-N bonds (Fig. 2). The deformation maps show aspherical ED features on $\mathrm{Zn}$ metal centres, i.e. a pronounced deviation from the expected spherical $3 \mathrm{~d}^{10}$ density of a $\mathrm{Zn}$ atom. The lone pair on the $\mathrm{N}$ atoms along the N-Zn-N plane (blue contours in 2D map and blue lumps in 3D maps) are polarized toward the charge concentration regions of the $\mathrm{Zn}$ atoms. In contrast to the $\mathrm{Zn}-\mathrm{N}$ bonding, there is a strong overlap between the $\mathrm{N}$ lone pairs and Co orbitals leading to ED accumulation in Co-N bonds. This suggests significant covalency in these bonds similar to the features observed in deformation densities of the $\mathrm{C}-\mathrm{N}$ and $\mathrm{C}-\mathrm{C}$ bonds in the imidazolate rings (Fig. S9).

To quantify the metal-ligand bonding we use topological analysis based on QTAIM theory, ${ }^{29}$ and we compare EDs obtained from experiments as well as single point periodic DFT calculations in the experimental geometry (Table 1 and Table S5). Although the bond path lengths (R) are indistinguishable between the two structures, the $\mathrm{Zn}-\mathrm{N}$ bonds have lower experimental rho $(\rho)$ values $\left(0.51-0.57 \mathrm{e}^{-3}\right)$ at the bond critical points $(\mathrm{BCPs})$ compared with the $\mathrm{Co}-\mathrm{N}$ bonds $\left(0.60-0.72 \mathrm{e}^{-3}\right)$. This corroborates the charge accumulation between Co and $\mathrm{N}$ observed in the static deformation maps (Fig. 2). The positive Laplacian values $\left(\nabla^{2} \rho\right)$ at the BCPs are typical of metal-ligand bonding ${ }^{30}$ but we do not observe a clear trend in the experimental values for the $\mathrm{Zn}-\mathrm{N}$ and $\mathrm{Co}-\mathrm{N}$ bonds presumably due to experimental noise (see supporting information, Fig. S2 and Fig. S4). The theoretical Laplacian values are consistently less positive in $\mathrm{Co}-\mathrm{N}$ bonds than $\mathrm{Zn}-\mathrm{N}$ bonds corroborating a more covalent character in the former. The local kinetic, potential and total energy densities evaluated at the BCPs characterize the metal-ligand interactions as a mixture of closed/shared shell interactions generally observed in transition metal coordination complexes ${ }^{30,31}$. The kinetic energy density per electron $\left(\mathrm{G}_{\mathrm{b}} / \rho\right)$ is slightly higher in the $\mathrm{Zn}-\mathrm{N}$ 
bonds, which corroborates their more ionic nature. Similarly, the larger $\mid \mathrm{V}_{\mathrm{b}} / \mathrm{G}_{\mathrm{b}}$ ratio and larger negative total energy density per electron $\left(\mathrm{H}_{\mathrm{b}} / \rho\right)$ at the BCPs show that the $\mathrm{Co}-\mathrm{N}$ bonds have more covalent character than the $\mathrm{Zn}-\mathrm{N}$ bonds. It is interesting to note that differences in the topological parameters $\left(\rho, \mathrm{G}_{\mathrm{b}} / \rho, \mid \mathrm{V}_{\mathrm{b}} / \mathrm{G}_{\mathrm{b}}, \mathrm{H}_{\mathrm{b}} / \rho\right)$ between $\mathrm{Zn}$-ZIF-zni and Co-ZIF-zni are more prominent in the experimental model as compared to the theoretical model, thereby underpinning the importance of experimental benchmark for testing theoretical studies of chemical bonding in extended structures ${ }^{32}$. In summary, the ED analysis establishes that the $\mathrm{Co}-\mathrm{N}$ bonds are more covalent than the $\mathrm{Zn}-\mathrm{N}$ bonds.

Further, we calculated the total density of states (DOS) and projected density of states (PDOS) of Zn-ZIF-zni and Co-ZIF-zni as shown in Fig. S11 and Fig. S12. In Zn-ZIF-zni, analysis of the individual atomic orbitals PDOS reveals that the valence bands (VB) near the Fermi level are largely consist of ligand atoms with negligible participation from the $\mathrm{Zn}$ atoms (Fig. S11). On the contrary, the Co $3 \mathrm{~d}$ orbitals below the Fermi level forms narrow and broad bands in Co-ZIF-zni (Fig. S12). The broad bonding bands due to clear mixing of $3 \mathrm{~d}$ states of $\mathrm{Co}$ atoms and $2 \mathrm{p}$ states of $\mathrm{C}, \mathrm{N}$ atoms in the $\mathrm{VB}$ region account for the partial covalent character of $\mathrm{Co}-\mathrm{N}$ bonds and also hint at the possibility of $\pi$-backbonding interactions between Co and imidazolate ligands.

The difference in chemical bonding impact the thermal vibrations of the metal and $\mathrm{N}$ atoms in the respective ZIFs. The evolution of atomic displacement parameters (ADP) with increasing temperature provide insights into the structural dynamics ${ }^{33}$ and therefore information relevant to the thermal stabilities of Zn/Co-ZIF-zni. We have collected synchrotron single crystal X-ray diffraction data for structural analysis for Zn-ZIF-zni and Co-ZIF-zni at temperatures of 25K, 100K, 200K, $300 \mathrm{~K}$ and 400 K. The mean equivalent isotropic ADPs of the metals $\left(<\mathrm{U}_{\mathrm{eq}}{ }^{\mathrm{M}}>\right)$, N atoms $\left(<\mathrm{U}_{\mathrm{eq}}{ }^{\mathrm{N}}>\right)$ and $\mathrm{C}$ atoms $\left(<\mathrm{U}_{\mathrm{eq}}{ }^{\mathrm{C}}>\right)$ 
are compared for all temperatures (Fig. S13). The Zn atoms have larger ADPs than the Co atoms reflecting the stiffer covalent $\mathrm{Co}-\mathrm{N}$ bonds, which restrict the movement of the Co atoms. Not surprisingly, the ADPs of the $\mathrm{N}$ and $\mathrm{C}$ atoms in the imidazolate rings are higher than for the metals (lighter mass). The equivalent isotropic ADPs $\left(\mathrm{U}_{\mathrm{eq}}\right)$ of ring $\mathrm{C}$ atoms (Fig. S14) and the rate of change of the ADPs with temperature (Fig. S15) indicate that the $\mathrm{C} 1$ and $\mathrm{C} 2$ atoms of ring 1 and $\mathrm{C} 4$ and $\mathrm{C} 5$ atoms of ring 2 display very pronounced out-of-plane libration similar to benzene ring in MOF-5. ${ }^{33}$ This reflects significant local distortion of the imidazolate ring with increasing temperature, where the upper part of the ring $(\mathrm{M} 1-\mathrm{N}-\mathrm{C}-\mathrm{N}-\mathrm{M} 2)$ vibrates less out-of-plane than the lower part $(-\mathrm{C}-\mathrm{C}-)$.

To probe the distortion of the frameworks at a molecular level, the ratio of $\mathrm{U}_{\text {eq }}$ values of ring $\mathrm{C}$ atom and its nearest neighbour metal atom $(\mathrm{Zn}, \mathrm{Co})$ is calculated as $\left.\mathrm{R}_{\mathrm{d}}=\mathrm{U}_{\mathrm{eq}}(\mathrm{Zn}, \mathrm{Co}) / \mathrm{U}_{\mathrm{eq}}(\mathrm{C})\right)$. The nitrogen atoms are excluded from this parameter because they are bonded to both $\mathrm{C}$ and $\mathrm{M}$ atoms and hence can introduce biases to this analysis. $R_{d}$ is an estimate of difference in thermal vibration between the metal atoms and the imidazolate rings, and it is an indirect measure of the framework distortion, see Fig. 3. Beyond $200 \mathrm{~K}$, the ADPs of the "lower ring" C1 and C2 increases strongly compared with Zn and Co atoms, whereas the ADP of the $\mathrm{C} 3$ atom bonded to two nitrogens largely follow the trend of the metal $\left(\mathrm{dU}\right.$ eq $(\mathrm{C} 3) / \mathrm{dT} \sim 1.9^{*} 10^{-4} \AA^{2} \mathrm{~K}^{-1}, \mathrm{dU}$ eq $(\mathrm{Zn}, \mathrm{Co}) / \mathrm{dT} \sim 1.4 * 10^{-4} \AA^{2} \mathrm{~K}^{-1}$, Fig. S16, Fig. S17). Comparison of $\mathrm{R}_{\mathrm{d}}$ values across all temperatures reveal larger difference in ADPs between the tightly bound Co and the flexible $\mathrm{C}$ atoms of ring 1 than for $\mathrm{Zn}$ and a similar trend is seen for the $\mathrm{Co}$ atom and the $\mathrm{C}$ atoms of the other imidazolate rings (Fig. S18, S19 and Fig. S20). Co-ZIF-zni clearly exhibits greater framework distortion during heating to $400 \mathrm{~K}$ and therefore have lower thermal stability than Zn-ZIF-zni ${ }^{9}$. 
It was not possible to study the structural changes beyond $400 \mathrm{~K}$ using single crystal X-ray crystallography due to deterioration in data quality. Instead, multi-temperature Raman spectroscopy was employed to indirectly follow structural changes. Sun et al. used temperature dependent Fourier transform infrared (FTIR) spectroscopy to study the thermolysis mechanism of ZIF-8 (Zn) and ZIF-67 (Co) $\mathrm{MOFs}^{34}$. Here Raman spectra were recorded from $300 \mathrm{~K}$ to $773 \mathrm{~K} / 823 \mathrm{~K}$ under nitrogen flow in the range of $1100-1600 \mathrm{~cm}^{-1}$ since signature peaks of $\mathrm{Zn}-\mathrm{N} / \mathrm{Co}-\mathrm{N}$ bonds were too weak to detect in the lower wavenumber region. The major peaks are assigned ${ }^{35}$ to the following modes in imidazolate ring- symmetric stretch of longer $\mathrm{C}-\mathrm{N}$ bonds $\left(1145-1177 \mathrm{~cm}^{-1}\right)$, symmetric stretch of shorter $\mathrm{C}-\mathrm{N}$ bonds in N-C$\mathrm{N}$ linkage $\left(1255-1284 \mathrm{~cm}^{-1}\right)$ and $\mathrm{C}=\mathrm{C}$ bond stretching $\left(1490-1495 \mathrm{~cm}^{-1}\right)$ (Fig. S21). The Raman peaks broaden with increasing temperature and the peak positions shift to lower wavenumbers because of lattice expansion. Beyond $700 \mathrm{~K}$, the peak intensities in Co-ZIF-zni drastically reduce and identification of the weaker $\mathrm{C}=\mathrm{C}$ Raman band becomes difficult.

The symmetric stretching modes $\left[v_{\mathrm{S}}(\mathrm{C} 1 \mathrm{~N} 1, \mathrm{C} 2 \mathrm{~N} 2), v_{\mathrm{S}}(\mathrm{C} 3 \mathrm{~N} 1, \mathrm{C} 3 \mathrm{~N} 2)\right]$ in the imidazolate rings of Co-ZIF-zni have lower frequencies than in Zn-ZIF-zni at all temperatures indicating weaker bonds in the former MOF (Fig. 3, ring 1 is a representative example for all imidazolate linkers). This observation emphasizes the implicit role of $\pi$-backbonding interaction between Co and ligands for weakening the imidazolate bonds of Co-ZIF-zni. The two characteristic C-N stretching modes in Zn-ZIF-zni exhibit a smooth red shift from $1176 \mathrm{~cm}^{-1}$ to $1163 \mathrm{~cm}^{-1}$ and from $1284 \mathrm{~cm}^{-1}$ to $1273 \mathrm{~cm}^{-1}$ during heating. In contrast, the shift of peak positions ( $1167 \mathrm{~cm}^{-1}$ to $1144 \mathrm{~cm}^{-1}, 1277 \mathrm{~cm}^{-1}$ to $\left.1255 \mathrm{~cm}^{-1}\right)$ is discontinuous in CoZIF-zni, particularly the $v_{\mathrm{S}}(\mathrm{C} 3 \mathrm{~N} 1, \mathrm{C} 3 \mathrm{~N} 2)$ modes show inflection beyond $673 \mathrm{~K}$. This suggests strong ring deformation prior to imidazolate bond dissociation in Co-ZIF-zni as the temperature approaches decomposition point (803 K, Fig. S1b). In Zn-ZIF-zni, the C-N Raman peaks are observed up to $823 \mathrm{~K}$ 
(Fig. S19), i.e. close to melting point $(\sim 860 \mathrm{~K})$, suggesting that the imidazolate rings remain intact in the melted form due to relatively stronger imidazolate bonds.

In order to further probe the role of the metal-N bonds on the thermal behavior of these ZIF compounds, we prepared a series of nine solid solution (SS) materials, $\mathrm{Co}_{\mathrm{x}} \mathrm{Zn}_{1-\mathrm{x}}-\mathrm{ZIF}-\mathrm{zni}$, where the Co mole fraction (x) ranges from 0.3 to as low as 0.003 . The final stoichiometry for each SS was determined from Inductively Coupled Plasma Optical Emission Spectrometry (ICP-OES) analysis (Table S11). TGA coupled with DSC measurements were done by heating the SS samples to $750{ }^{\circ} \mathrm{C}$ in argon to analyze their thermal behavior. The phase diagram for all SS materials is presented in Fig. 4 based on DSC-TGA measurements. $\mathrm{T}_{\mathrm{m}}$ is the offset temperature of the endothermic peak in DSC profile, while $\mathrm{T}_{\mathrm{d}}$ is the onset decomposition temperature deduced from TGA graphs (Fig. S22).

We observe a systematic dependence of thermal stability of $\mathrm{Co}_{\mathrm{x}} \mathrm{Zn}_{1-\mathrm{x}}$-ZIF-zni SS phases on percentage of Co doping in the crystal structure (Fig. 4). In the mole fraction range of 0.3-0.04 Co, $\mathrm{T}_{\mathrm{d}}$ is lower than $\mathrm{T}_{\mathrm{m}}\left(\sim 7-21^{\circ} \mathrm{C}\right)$ suggesting decomposition for these SS materials. Thus, even a very low quantity of Co dopant $(\sim 4 \%)$ fundamentally alters the thermal stability with respect to the parent Zn-ZIF-zni structure. At ultralow mole fractions of $\mathrm{Co}(\mathrm{x}=0.01$ to 0.003$)$ in $\mathrm{Co}_{\mathrm{x}} \mathrm{Zn}_{1-\mathrm{x}}-\mathrm{ZIF}-\mathrm{zni}$, sharp endothermic peaks of melting are observed as the corresponding $\mathrm{T}_{\mathrm{m}}$ values are lower than $\mathrm{T}_{\mathrm{d}}$ values by $\sim 11{ }^{\circ} \mathrm{C}$. The melting behavior is observed macroscopically where the melted SS compounds lose their needle-like morphology compared with decomposed SS samples (Fig. 4). It is intriguing that a few percent of Co atoms in Zn-ZIF-zni is able to disrupt the lattice and cause decomposition. Hence, in a cluster of $3 \times 3 \times$ $3 \mathrm{Zn}$ atoms in the Zn-ZIF-zni lattice mimicking 4\% doped Co SS phase, substitution of one Zn by Co causes chemical disruption and decomposition (Fig. S23). This imply that the "correlation length" of the local chemical bonding consisting of at least a trimer of metal atoms extends for about 8-11 Å in Zn-ZIF- 
zni. To rephrase it, a theoretical cluster calculation would not represent the Zn-ZIF-zni chemical bonding if not containing at least $4 \times 4 \times 4 \mathrm{Zn}$ atoms.

In summary, using synchrotron X-ray diffraction we have determined the EDs of a meltable MOF, Zn-ZIF-zni and the isostructural Co-ZIF-zni, which decomposes upon heating. Static ED deformation maps and topological analysis reveal that for Co-ZIF-zni, the $\mathrm{Co}-\mathrm{N}$ bonds have distinct covalent features, whereas the $\mathrm{Zn}-\mathrm{N}$ bonds in $\mathrm{Zn}-\mathrm{ZIF}-\mathrm{zni}$ are more ionic. This is furthermore corroborated by band structure calculations, which shows covalent mixing between the valence states of $\mathrm{C}, \mathrm{N}$ atoms and the $3 \mathrm{~d}$ orbitals of Co atoms in Co-ZIF-zni. Temperature dependent Raman spectroscopy shows that the imidazolate bonds in Zn-ZIF-zni are relatively more stable than in Co-ZIF-zni, which show an abrupt bond weakening above $673 \mathrm{~K}$. The inherently weaker imidazolate bonds in Co-ZIF-zni could be related to subtle $\pi$-backbonding between Co and imidazolate ligands. Multi-temperature X-ray diffraction data corroborate that the thermally induced framework distortion is smaller in Zn-ZIF-zni compared with CoZIF-zni. The thermal decomposition versus melting behavior was studied in a series of Co-substituted Zn-ZIF-zni solid solution phases. Even a very low Co concentration of $\sim 4 \%$ leads to disruption of the $\mathrm{Zn}$-ZIF crystal lattice causing a fundamental change in its thermal behavior. It appears that the relatively more ionic $\mathrm{Zn}-\mathrm{N}$ bond and more stable imidazolate linkers facilitate melting in Zn-ZIF-zni. In contrast, the more directional covalent $\mathrm{Co}-\mathrm{N}$ bonding, and resultant weakened imidazolate bonding, leads to decomposition in Co-ZIF-zni. This shows that the nature of the metal-ligand bonds as well as the strengths of internal bonds in the linker molecule, play a key role in controlling the delicate balance between melting and decomposition process in this class of ZIF compounds. There appears to be considerable chemical space for example through ligand functionalization or choice of metal to design new MOF glasses.

\section{Methods}


Sample synthesis. Single crystals of Zn-ZIF-zni, Co-ZIF-zni and solid solution Cox $\mathrm{Zn}_{1-\mathrm{x}}$-ZIF-zni (x= 0.4-0.003) were synthesized by autoclave synthesis.

$0.5 \mathrm{mmol}(148.75 \mathrm{mg})$ of $\mathrm{Zn}\left(\mathrm{NO}_{3}\right)_{2} \cdot 6 \mathrm{H}_{2} 0$ and $4 \mathrm{mmol}(272.31 \mathrm{mg})$ of Imidazole was dissolved in $9 \mathrm{ml}$ DMF and $6 \mathrm{ml}$ butanol. The resulting solution was transferred to a glass vial kept inside a $25 \mathrm{ml}$ Teflon coated steel autoclave. The autoclave was closed and heated at $150^{\circ} \mathrm{C}$ for 5 days. White needle shaped crystals were obtained and washed with ethanol 3 times.

$0.5 \mathrm{mmol}(124.52 \mathrm{mg})$ of $\mathrm{Co}(\mathrm{OAc})_{2} .4 \mathrm{H}_{2} \mathrm{O}$ and $1 \mathrm{mmol}(68.08 \mathrm{mg})$ of Imidazole was added to $5 \mathrm{ml}$ of quinoline and $5 \mathrm{ml}$ of isoamyl alcohol and stirred for 12 hours at room temperature. The resulting mixture was transferred to a glass vial kept inside a $25 \mathrm{ml}$ Teflon coated steel autoclave. The autoclave was closed and heated at $140^{\circ} \mathrm{C}$ for 3 days. Purple blocked shaped crystals were obtained and washed with ethanol 3 times.

Nine solid solutions $\mathrm{Co}_{\mathrm{x}} \mathrm{Zn}_{1-\mathrm{x}}$-ZIF-zni single crystals were synthesized following the procedure for the preparation of pristine $\mathrm{Zn}-\mathrm{ZIF}-\mathrm{zni}$. The precursor metal salts- $\mathrm{Zn}\left(\mathrm{NO}_{3}\right)_{2} \cdot 6 \mathrm{H}_{2} \mathrm{O}$ and $\mathrm{Co}\left(\mathrm{NO}_{3}\right)_{2} \cdot 6 \mathrm{H}_{2} \mathrm{O}$ were taken in the following stoichiometric ratios-3:1, 4:1, 6:1, 8:1, 12:1, 20:1, 50:1, 100:1. 200:1.

Single-crystal synchrotron X-ray diffraction. Single crystals synchrotron X-ray diffraction data were measured on Zn-ZIF-zni and Co-ZIF-zni at beamline BL02B1 at SPring-8, Japan. A PILATUS3 X CdTe $1 \mathrm{M}$ photon counting detector was used to collect the diffracted intensities at $\lambda=0.24823(5) \AA$ and at $\mathrm{T}=25 \mathrm{~K}$ using Helium cryostat. The crystal to detector distance was kept at $130 \mathrm{~mm}$. For both crystals, 1800 frames were collected at four different $\varphi$ angles: $0^{\circ},-15^{\circ},-30^{\circ}$ and $-45^{\circ}$ with $\omega$ scans $\left(0^{\circ}\right.$ to $\left.180^{\circ}\right)$ and fixed $2 \theta=0^{\circ}$. Scan width for $\omega$ scan was $0.1^{\circ}$ per frame with an exposure time of 0.8 seconds per $0.1^{\circ}$ 
scan. The frames was integrated using SAINT in which the integration box sizes were optimized manually by careful inspection of each run. The data were scaled in the program SADABS, ${ }^{36}$ which was also used to carry out an absorption and oblique correction. Finally, data averaging was performed with the program SORTAV. ${ }^{37}$ A completeness of more than $99.5 \%$ was obtained up to $1.35 \AA^{-1}$ and $1.25 \AA^{-1}$ for Zn-ZIF-zni and Co-ZIF-zni, respectively, and data redundancies of $22-25$. Two low order reflections (200) and (400) were omitted from the refinement. The (200) reflection was hidden behind the beamstop while the (400) reflection was overexposed. The crystal structures were solved with the SHELXT program $^{38}$ and refined by SHELXL ${ }^{39}$ included in WINGX package. The hydrogen atom was located in the difference Fourier map and its position and isotropic ADPs were refined.

Multi-Temperature crystallographic data. Single crystal synchrotron X-ray diffraction data were collected at $100 \mathrm{~K}, 200 \mathrm{~K}, 300 \mathrm{~K}$ and $400 \mathrm{~K}$ for Zn-ZIF-zni and Co-ZIF-zni using the same crystals as for the $25 \mathrm{~K}$ high-resolution ED data sets. The crystal to detector distance was kept at $130 \mathrm{~mm}$. For all data sets, 1800 frames were collected at two different $\varphi$ angles: $0^{\circ}$ and $-45^{\circ}$ with $\omega$ scans $\left(0^{\circ}\right.$ to $\left.180^{\circ}\right)$ and fixed $2 \theta=0^{\circ}$. The scan width for $\omega$ scan was $0.1^{\circ}$ per frame with an exposure time of 0.8 seconds. The frames were integrated using SAINT and the data were scaled using SADABS which was also used to carry out an absorption and oblique correction. Data averaging was performed with the program SORTAV. The hydrogen atoms were geometrically fixed using riding model constraints. The higher thermal motions (manifested as large ADPs) of the imidazolate ring 1 and ring 2 atoms in $400 \mathrm{~K}$ crystal structures of both Zn-ZIF-zni and Co-ZIF-zni were modeled in two different ways: i) an ordered model, in which the ring atoms are represented as elongated ellipsoids (Fig. S8), and ii) a disordered model, in which the ring atoms are split into two components occupying different crystallographic sites. 
Multipole modelling and electron density analysis. The ED was obtained from the experimental structure factors using the Hansen-Coppens multipole formalism ${ }^{27}$ in the program package XD2015. ${ }^{40}$ The refinements were carried out with scattering factors derived from Slater-type orbital (STO) atomic relativistic wavefunctions found in the SCM data bank of XD2015. In the refinement strategy, experimental weights and a $\mathrm{I} / \sigma(\mathrm{I})>3$ were used against $\mathrm{F}^{2}$. Further details about the multipole refinement is provided in the supporting information. The quantitative analysis of the electron density topology was performed using the XDPROP program in XD2015 with the kinetic energy density G estimated using the Abramov functional. ${ }^{41}$ The potential energy density $\mathrm{V}$ and the total energy density $\mathrm{H}$ were calculated using the local virial theorem.

Theoretical calculations. Positional parameters obtained from the multipolar refinement were used for the density functional theory calculations using the Perdew, Burke, and Ernzerhof (PBE) GGA functional $^{42}$ with $\mathrm{TZVP}^{43}$ basis set for Zn, Co, C, N, H atoms included in CRYSTAL17 package. ${ }^{44}$ To avoid issues related to quasi-linear dependence, the coefficients of the outermost $\mathrm{s}$ and $\mathrm{p}$ functions were recalculated and the f functions were removed. The shrinking factors (IS1, IS2, and IS3) and the reciprocal lattice vectors were set to 4 (with 13 k-points in the irreducible Brillouin zone). The bi-electronic Coulomb and exchange series values for the truncation parameter were set as ITOL1-ITOL4 $=7$ and ITOL5 $=14$, respectively, for the calculations. Both antiferromagnetic (AFM) and ferromagnetic $(\mathrm{FM})$ spin ordering was considered for SCF calculation of Co-ZIF-zni. Our results revealed that the AFM state is energetically more favorable than the FM state by $230 \mathrm{meV}$ for Co-ZIF-zni and therefore all the calculations were performed on AFM phase of Co-ZIF-zni. In case of Zn-ZIF-zni, the level shifter was set to 0.6 Hartree/cycle with $30 \%$ of Fock/KS matrices mixing. In Co-ZIF-zni, the level shifter was set to 0.7 Hartree/cycle with $50 \%$ of Fock/KS matrices mixing. An SCF convergence limit of the order of $10^{-7}$ 
Hartree was used. Upon convergence, the periodic electron densities as obtained from the wave function was subjected to the topological analysis by Bader's Quantum Theory of Atoms in Molecules (QTAIM) ${ }^{29}$ using the TOPOND program, which is directly interfaced with the CRYSTAL17 code. For computations of total density of states and individual atomic orbital projected density of states of the metal atoms and $\mathrm{C}, \mathrm{N}$ atoms, the shrinking factors (IS1, IS2, and IS3) and the reciprocal lattice vectors were set to 14 (with $244 \mathrm{k}$-points in the irreducible Brillouin zone).

Raman spectroscopy. Multi-temperature Raman spectra were recorded on a Renishaw inVia Reflex Micro Raman spectrometer. In case of Zn-ZIF-zni, the single crystal was exposed to $785 \mathrm{~nm} 50 \mathrm{~mW}$ He laser operated at $10 \%$ power and 1200 lines/mm grating. The exposure time was $1 \mathrm{sec}$ and the number of accumulations was 30/40. For Co-ZIF-zni, the single crystal was exposed to $457 \mathrm{~nm} 9.5 \mathrm{~mW}$ Ar-ion laser operated at $50 \%$ power and 2400 lines/mm grating. The exposure times were $1-2$ sec and the number of accumulations was 30. The temperature dependent Raman studies were done using a Linkam THMS 600 heating-cooling stage. Temperature was monitored through the temperature controller attached to the Linkam stage. All the respective spectra were collected under nitrogen flow. The Raman spectra obtained were smoothened using the Savitsky-Golay algorithm with a polynomial order of two and baseline correction was done to remove the background.

Differential Scanning Calorimetry and Thermogravimetric analysis. For these experiments, a NETZSCH DSC 404 F1 Pegasus instrument was used. All the experiments with a heating rate of 10 $\mathrm{K} / \mathrm{min}$ were conducted in an argon atmosphere. Instrumental contributions were subtracted by measuring a correction file on an empty, clean sample crucible using an identical heating regime measured immediately before each sample. 
Inductively Coupled Plasma Optical Emission Spectrometry (ICP-OES). Samples of $\mathrm{Co}_{\mathrm{x}} \mathrm{Zn}_{1-\mathrm{x}}$-ZIFzni solid solution were dissolved with PlasmaPure Nitric acid $\left(\mathrm{HNO}_{3}\right)$ and diluted to $1 \%$ acid concentration with MilliQ-water. The measurements were carried out on a Spectro ARCOS ICP-OES equipped with a Burgener Nebulizer and Cyclonic Spray Chamber with an ASX-520 Auto sampler. For quantification of the elements, standard curves series waere measured ${ }^{45}$. The series consisted of concentrations for each elements: $0,0.01,0.05,0.1,0.5,1,5,10,20,50 \mathrm{ppm}$.

\section{References}

1. Furukawa H, Cordova KE, O'Keeffe M, Yaghi OM. The Chemistry and Applications of MetalOrganic Frameworks. Science 2013, 341(6149): 1230444.

2. Guillerm V, Kim D, Eubank JF, Luebke R, Liu X, Adil K, et al. A supermolecular building approach for the design and construction of metal-organic frameworks. Chem Soc Rev 2014, 43(16): 6141-6172.

3. Bennett TD, Cheetham AK. Amorphous Metal-Organic Frameworks. Acc Chem Res 2014, 47(5): 1555-1562.

4. Orellana-Tavra C, Baxter EF, Tian T, Bennett TD, Slater NKH, Cheetham AK, et al. Amorphous metal-organic frameworks for drug delivery. Chem Commun 2015, 51(73): 13878-13881.

5. Wu X, Yue H, Zhang Y, Gao X, Li X, Wang L, et al. Packaging and delivering enzymes by amorphous metal-organic frameworks. Nat Commun 2019, 10(1): 5165.

6. Fonseca J, Choi S. Flexible amorphous metal-organic frameworks with $\pi$ Lewis acidic pore surface for selective adsorptive separations. Dalton Trans 2021, 50(9): 3145-3154.

7. Bennett TD, Goodwin AL, Dove MT, Keen DA, Tucker MG, Barney ER, et al. Structure and Properties of an Amorphous Metal-Organic Framework. Phys Rev Lett 2010, 104(11): 115503. 
8. Bennett TD, Simoncic P, Moggach SA, Gozzo F, Macchi P, Keen DA, et al. Reversible pressureinduced amorphization of a zeolitic imidazolate framework (ZIF-4). Chem Commun 2011, 47(28): 7983-7985.

9. Healy C, Patil KM, Wilson BH, Hermanspahn L, Harvey-Reid NC, Howard BI, et al. The thermal stability of metal-organic frameworks. Coord Chem Rev 2020, 419: 213388.

10. Bennett TD, Horike S. Liquid, glass and amorphous solid states of coordination polymers and metal-organic frameworks. Nat Rev Mater 2018, 3(11): 431-440.

11. Wang Y, Jin H, Ma Q, Mo K, Mao H, Feldhoff A, et al. A MOF Glass Membrane for Gas Separation. Angew Chem Int Ed 2020, 59(11): 4365-4369.

12. Frentzel-Beyme L, Kloß M, Kolodzeiski P, Pallach R, Henke S. Meltable Mixed-Linker Zeolitic Imidazolate Frameworks and Their Microporous Glasses: From Melting Point Engineering to Selective Hydrocarbon Sorption. J Am Chem Soc 2019, 141(31): 12362-12371.

13. Zhou C, Longley L, Krajnc A, Smales GJ, Qiao A, Erucar I, et al. Metal-organic framework glasses with permanent accessible porosity. Nat Commun 2018, 9(1): 5042.

14. Ali MA, Ren J, Zhao T, Liu X, Hua Y, Yue Y, et al. Broad Mid-Infrared Luminescence in a Metal-Organic Framework Glass. ACS Omega 2019, 4(7): 12081-12087.

15. Ali MA, Liu X, Li Y, Ren J, Qiu J. Nonlinear-Optical Response in Zeolitic Imidazolate Framework Glass. Inorg Chem 2020, 59(12): 8380-8386.

16. Jiang G, Qu C, Xu F, Zhang E, Lu Q, Cai X, et al. Glassy Metal-Organic-Framework-Based Quasi-Solid-State Electrolyte for High-Performance Lithium-Metal Batteries. Adv Funct Mater 2021, n/a(n/a): 2104300-2104309.

17. Widmer RN, Lampronti GI, Kunz B, Battaglia C, Shepherd JH, Redfern SAT, et al. Manufacturing Macroporous Monoliths of Microporous Metal-Organic Frameworks. ACS Appl Nano Mater 2018, 1(2): 497-500.

18. Bennett TD, Tan J-C, Yue Y, Baxter E, Ducati C, Terrill NJ, et al. Hybrid glasses from strong and fragile metal-organic framework liquids. Nat Commun 2015, 6(1): 8079. 
19. Gaillac R, Pullumbi P, Beyer KA, Chapman KW, Keen DA, Bennett TD, et al. Liquid metalorganic frameworks. Nat Mater 2017, 16(11): 1149-1154.

20. Bennett TD, Keen DA, Tan J-C, Barney ER, Goodwin AL, Cheetham AK. Thermal Amorphization of Zeolitic Imidazolate Frameworks. Angew Chem Int Ed 2011, 50(13): 30673071.

21. Frentzel-Beyme L, Kloß M, Pallach R, Salamon S, Moldenhauer H, Landers J, et al. Porous purple glass - a cobalt imidazolate glass with accessible porosity from a meltable cobalt imidazolate framework. J Mater Chem A 2019, 7(3): 985-990.

22. Hou J, Ríos Gómez ML, Krajnc A, McCaul A, Li S, Bumstead AM, et al. Halogenated MetalOrganic Framework Glasses and Liquids. J Am Chem Soc 2020, 142(8): 3880-3890.

23. Tolborg K, Iversen BB. Electron Density Studies in Materials Research. Chem Eur J 2019, 25(66): 15010-15029.

24. Poulsen RD, Bentien A, Graber T, Iversen BB. Synchrotron charge-density studies in materials chemistry: $16 \mathrm{~K}$ X-ray charge density of a new magnetic metal-organic framework material, [Mn2(C8H4O4)2(C3H7NO)2]. Acta Cryst A 2004, 60(5): 382-389.

25. Poulsen RD, Jørgensen MRV, Overgaard J, Larsen FK, Morgenroth W, Graber $\mathrm{T}$, et al. Synchrotron X-Ray Charge-Density Study of Coordination Polymer [Mn(HCOO)2(H2O)2]. Chem Eur J 2007, 13(35): 9775-9790.

26. Clausen HF, Overgaard J, Chen YS, Iversen BB. Synchrotron X-ray Charge Density Study of Coordination Polymer Co3(C8H4O4)4(C4H12N)2(C5H11NO)3 at 16 K. J Am Chem Soc 2008, 130(25): 7988-7996.

27. Hansen NK, Coppens P. Testing aspherical atom refinements on small-molecule data sets. Acta Crystallogr, Sect A: Cryst Phys,Diffr, Theor Gen Crystallogr 1978, 34(6): 909-921.

28. Zhou C, Stepniewska M, Sørensen JM, Scarpa L, Magnacca G, Boffa V, et al. Polymorph formation for a zeolitic imidazolate framework composition - $\mathrm{Zn}(\mathrm{Im}) 2$. Microporous Mesoporous Mater 2018, 265: 57-62. 
29. Bader R. Atoms in Molecules - A Quantum Theory, vol. 22 of International Series of Monographs in Chemistry. . Oxford, UK: Oxford University Press; 1990.

30. Macchi P, Sironi A. Chemical bonding in transition metal carbonyl clusters: complementary analysis of theoretical and experimental electron densities. Coord Chem Rev 2003, 238-239: 383412.

31. Gatti C. Chemical bonding in crystals: new directions. Z Kristallogr Cryst Mater 2005, 220(56): 399-457.

32. Kasai H, Tolborg K, Sist M, Zhang J, Hathwar VR, Filsø MØ, et al. X-ray electron density investigation of chemical bonding in van der Waals materials. Nat Mater 2018, 17(3): 249-252.

33. Lock N, Wu Y, Christensen M, Cameron LJ, Peterson VK, Bridgeman AJ, et al. Elucidating Negative Thermal Expansion in MOF-5. J Phys Chem C 2010, 114(39): 16181-16186.

34. Wu C, Xie D, Mei Y, Xiu Z, Poduska KM, Li D, et al. Unveiling the thermolysis natures of ZIF8 and ZIF-67 by employing in situ structural characterization studies. Phys Chem Chem Phys 2019, 21(32): 17571-17577.

35. Hashimoto S, Ono K, Takeuchi H, Harada I. Vibrational analysis of the imidazolate ring. Spectrochim Acta A Mol Biomol Spectrosc 1994, 50(8): 1647-1660.

36. Krause L, Herbst-Irmer R, Sheldrick GM, Stalke D. Comparison of silver and molybdenum microfocus X-ray sources for single-crystal structure determination. J Appl Crystallogr 2015, 48(1): 3-10.

37. Blessing R. Outlier Treatment in Data Merging. J Appl Crystallogr 1997, 30(4): 421-426.

38. Sheldrick G. SHELXT - Integrated space-group and crystal-structure determination. Acta Cryst A 2015, 71(1): 3-8.

39. Sheldrick G. Crystal structure refinement with SHELXL. Acta Cryst C 2015, 71(1): 3-8.

40. Volkov A, Macchi P, Farrugia L, Gatti C, Mallinson P, Richter T, et al. XD2015- a computer program package for multipole refinement, topological analysis of charge densities and 
evaluation of intermolecular energies from experimental and theoretical structure factors University at Buffalo, State University of New York, NY, USA; 2015.

41. Abramov Y. On the Possibility of Kinetic Energy Density Evaluation from the Experimental Electron-Density Distribution. Acta Cryst A 1997, 53(3): 264-272.

42. Perdew JP, Burke K, Ernzerhof M. Generalized Gradient Approximation Made Simple. Phys Rev Lett 1996, 77(18): 3865-3868.

43. Vilela Oliveira D, Laun J, Peintinger MF, Bredow T. BSSE-correction scheme for consistent gaussian basis sets of double- and triple-zeta valence with polarization quality for solid-state calculations. J Comput Chem 2019, 40(27): 2364-2376.

44. Dovesi R, Erba A, Orlando R, Zicovich-Wilson CM, Civalleri B, Maschio L, et al. Quantummechanical condensed matter simulations with CRYSTAL. WIREs Comput Mol Sci 2018, 8(4): e1360.

45. Harris DC. Quantitative chemical analysis., 8th edn. New York: W.H. Freeman and Co, 2010.

46. Turner MJ, McKinnon JJ, Jayatilaka D, Spackman MA. Visualisation and characterisation of voids in crystalline materials. CrystEngComm 2011, 13(6): 1804-1813. 


\section{Author information}

Sounak Sarkar, Thomas Bjørn Egede Grønbech, Aref Mamakhel, Martin Bondesgaard, Bo B. Iversen

${ }^{1}$ Center for Materials Crystallography, Department of Chemistry and Interdisciplinary Nanoscience Center (iNANO), Aarhus University, Langelandsgade 140, Aarhus DK-8000, Denmark.

Kunihisa Sugimoto

${ }^{2}$ Japan Synchrotron Radiation Research Institute (JASRI), 1-1-1 Kouto, Sayo-cho, Sayo-gun, Hyogo 679-5198, Japan.

Eiji Nishibori

${ }^{3}$ Faculty of Pure and Applied Sciences, Tsukuba Research Center for Energy Materials Science (TREMS), University of Tsukuba, 1-1-1 Tennodai, Tsukuba, Ibaraki 305-8571, Japan. 
Table 1. Topological analysis at bond critical points (BCPs) of metal-N bonds from experimental multipole model (first line) and periodic DFT calculations (second line in italics). $\mathrm{R}$ is the bond path length, which is a gradient trajectory length originating at the BCP and terminating at the nuclei (Fig. S10). $\rho$ and $\nabla^{2} \rho$ are the electron density and its Laplacian, respectively. $G_{b}, V_{b}$ and $H_{b}$ are the kinetic, potential and total energy density $\left(H_{b}=G_{b}+V_{b}\right)$ at the $B C P$, respectively. $\mathrm{H}_{b} / \rho$ and $\mathrm{G}_{b} / \rho$ are in atomic units (a.u.). $\varepsilon$ is the bond ellipticity $\left[\varepsilon=\left(\lambda_{1} / \lambda_{2}\right)-1\right]$ defined as the ratio of the eigenvalues $\lambda_{1}$ and $\lambda_{2}$ of the Hessian matrix that corresponds to the negative curvature of electron density along two perpendicular directions to the bond path.

\begin{tabular}{cccccc|cc}
\hline Bonds & $\mathrm{R}(\AA)$ & $\rho\left(\mathrm{e}^{-3}\right)$ & $\nabla^{2} \rho\left(\mathrm{e}^{-5}\right)$ & $\varepsilon$ & $\mathrm{G}_{\mathrm{b}} / \rho$ & $\mathrm{V}_{\mathrm{b}} \mid / \mathrm{G}_{\mathrm{b}}$ & $\mathrm{H}_{\mathrm{b}} / \rho$ \\
\hline \multirow{2}{*}{ Zn1-N4 } & 1.991 & 0.533 & 9.526 & 0.08 & 1.36 & 1.08 & -0.11 \\
& 1.991 & 0.606 & 8.812 & 0.06 & 1.26 & 1.19 & -0.24 \\
\hline \multirow{2}{*}{ Co1-N4 } & 1.994 & 0.678 & 9.808 & 0.11 & 1.30 & 1.22 & -0.28 \\
& 1.993 & 0.619 & 8.362 & 0.07 & 1.22 & 1.22 & -0.27 \\
\hline \multirow{2}{*}{ Zn1-N6 } & 1.987 & 0.512 & 9.808 & 0.09 & 1.41 & 1.05 & -0.07 \\
& 1.986 & 0.612 & 8.931 & 0.05 & 1.26 & 1.19 & -0.24 \\
\hline \multirow{2}{*}{ Co1-N6 } & 1.987 & 0.714 & 10.493 & 0.09 & 1.33 & 1.22 & -0.30 \\
& 1.987 & 0.626 & 8.595 & 0.03 & 1.23 & 1.22 & -0.27 \\
\hline \multirow{2}{*}{ Zn2-N3 } & 1.995 & 0.528 & 9.961 & 0.08 & 1.41 & 1.06 & -0.08 \\
& 1.995 & 0.602 & 8.746 & 0.06 & 1.25 & 1.19 & -0.23 \\
\hline \multirow{2}{*}{ Co2-N3 } & 1.990 & 0.719 & 9.669 & 0.09 & 1.27 & 1.26 & -0.33 \\
& 1.989 & 0.624 & 8.393 & 0.08 & 1.22 & 1.22 & -0.27 \\
\hline \multirow{2}{*}{ Zn2-N7 } & 1.979 & 0.564 & 10.471 & 0.09 & 1.42 & 1.08 & -0.12 \\
& 1.979 & 0.622 & 9.184 & 0.05 & 1.28 & 1.19 & -0.24 \\
\hline \multirow{2}{*}{ Co2-N7 } & 1.987 & 0.673 & 10.652 & 0.09 & 1.36 & 1.18 & -0.25 \\
& 1.981 & 0.635 & 8.732 & 0.05 & 1.25 & 1.22 & -0.27 \\
\hline
\end{tabular}




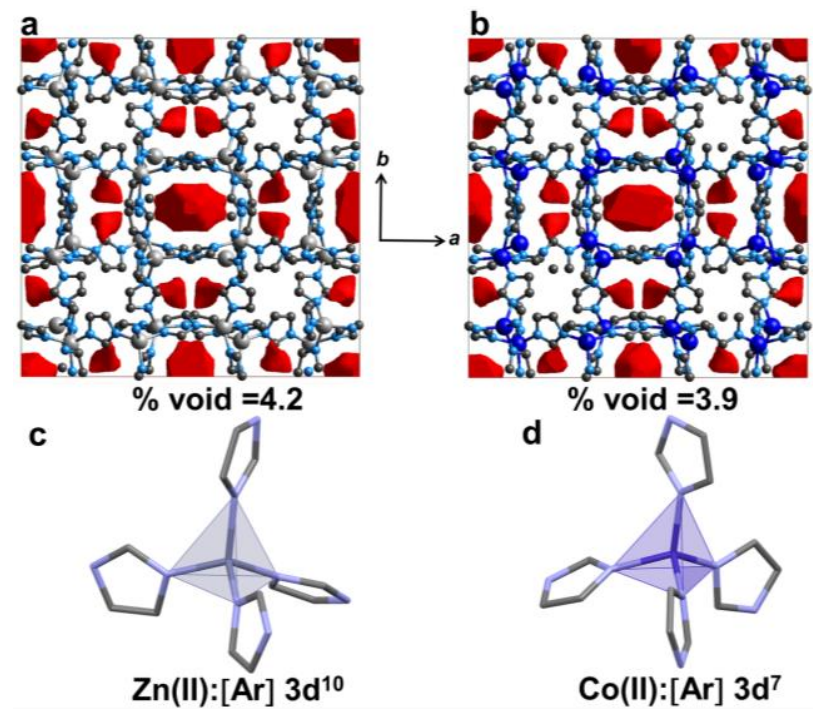

Fig. 1 | Structure of ZIF-zni MOFs. a-b, (Top) Unit cell packing with voids ${ }^{46}$ (red surfaces) viewed along the $c$ axis of unit cell in (a) Zn-ZIF-zni and (b) Co-ZIF-zni. c-d, Building unit of ZIF-zni with (c) Zn (II) and (d) Co (II) in a tetrahedral coordination with four crystallographic independent imidazolate rings. 


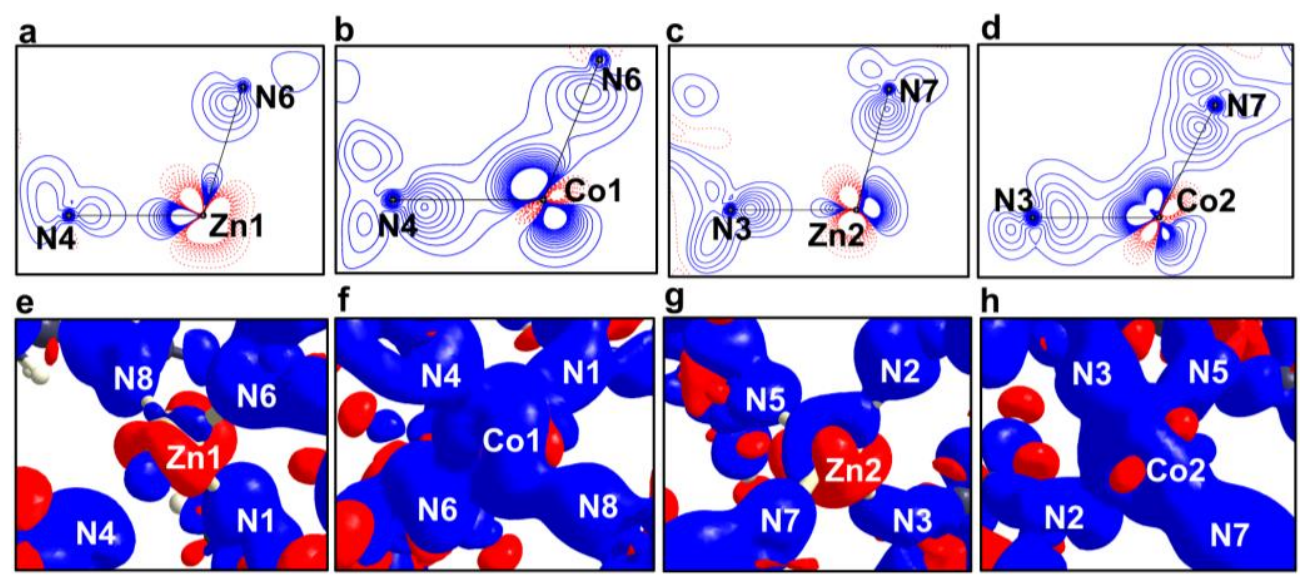

Fig. 2 | Static deformation maps from multipole modelling of experimental structure factors. a-d, 2D static deformation density in the plane of N-M-N for (a, c) Zn-ZIF-zni, (b, d) Co-ZIF-zni. Blue solid lines and red dots represents positive and negative contours, respectively, and the contour level is $\pm 0.1 \mathrm{e} \AA^{-3}$. e-h, 3D static deformation density of $\mathrm{MN}_{4}$ tetrahedra for (e, g) Zn-ZIF-zni, (f, h) Co-ZIF-zni. Blue regions indicates charge concentration region while red regions indicate charge depleted regions and the contour level is $\pm 0.1 \mathrm{e}^{-3}$. 

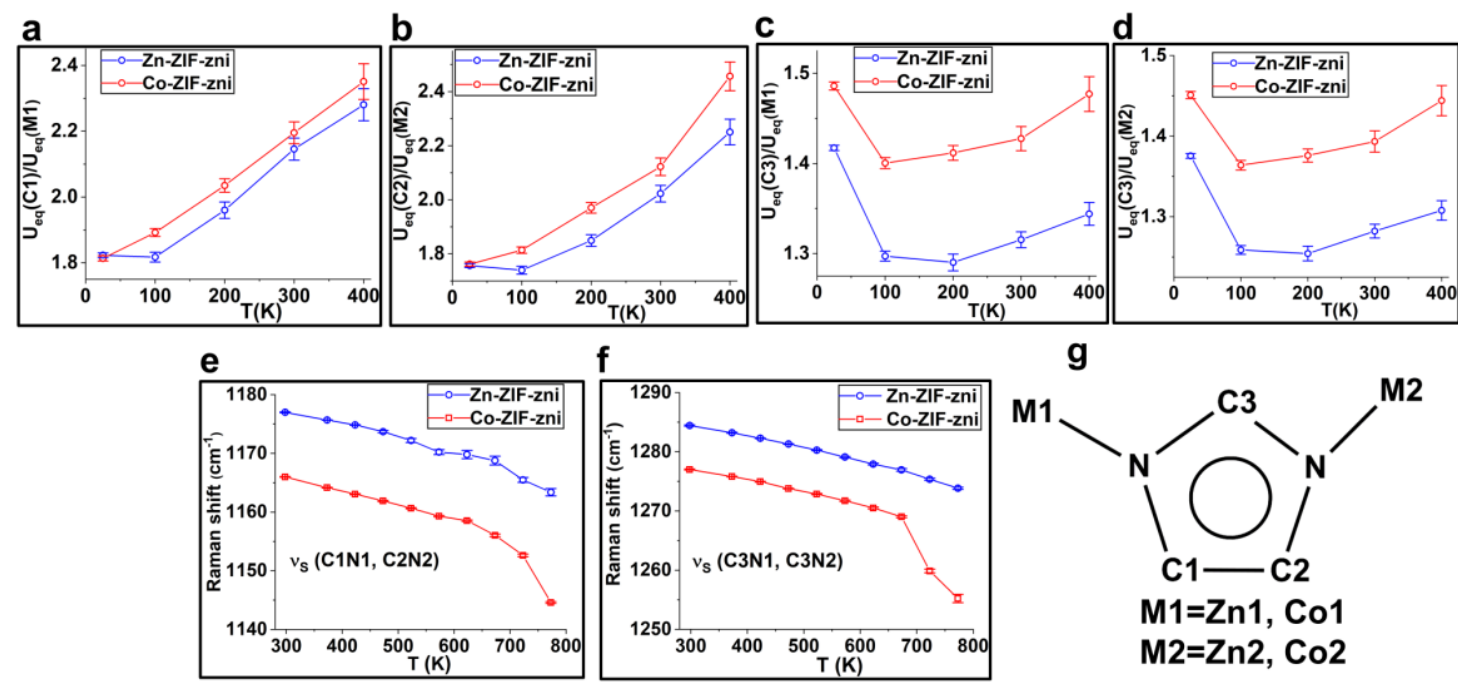

Fig. 3 | Structural variation of Zn-ZIF-zni and Co-ZIF-zni with temperature. a-d Comparison of temperaturedependent variation in $\mathrm{R}_{\mathrm{d}}$ (ratio of $\mathrm{U}_{\mathrm{eq}}$ values of $\mathrm{C}$ atoms of ring $1 \mathrm{~g}$ ) and its nearest neighbour $\mathrm{Zn} / \mathrm{Co}$ ) based on variable temperature synchrotron X-ray diffraction data. e-f. Comparison of temperature-dependent variation in Raman peak shifting corresponding to (e) symmetric stretch of longer $\mathrm{C}-\mathrm{N}$ bonds $\left(1145-1177 \mathrm{~cm}^{-1}\right)$ and (f) symmetric stretch of shorter C-N bonds in N-C-N linkage (1255-1284 $\left.\mathrm{cm}^{-1}\right)$ of imidazolate rings. $\mathbf{g}$, Molecular diagram of ring 1 bonded with respective metals as a representative example for all imidazolate linkers. 


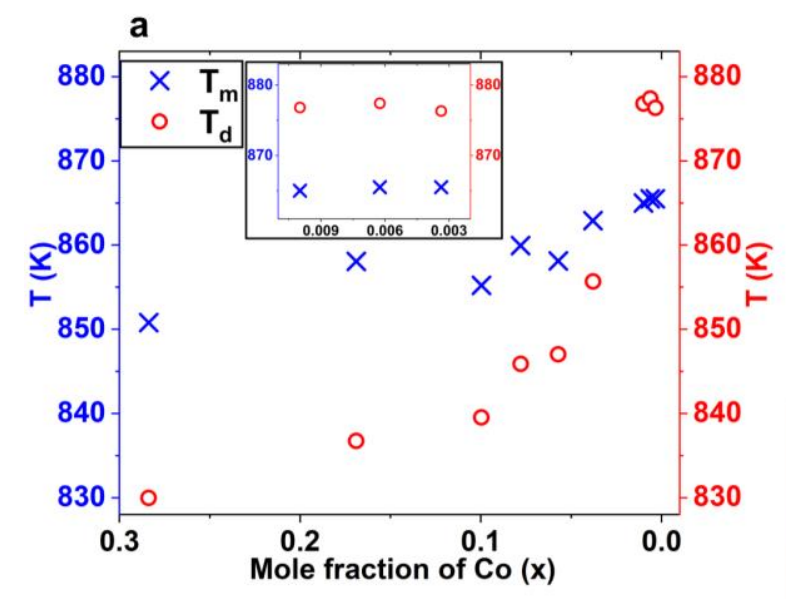

b

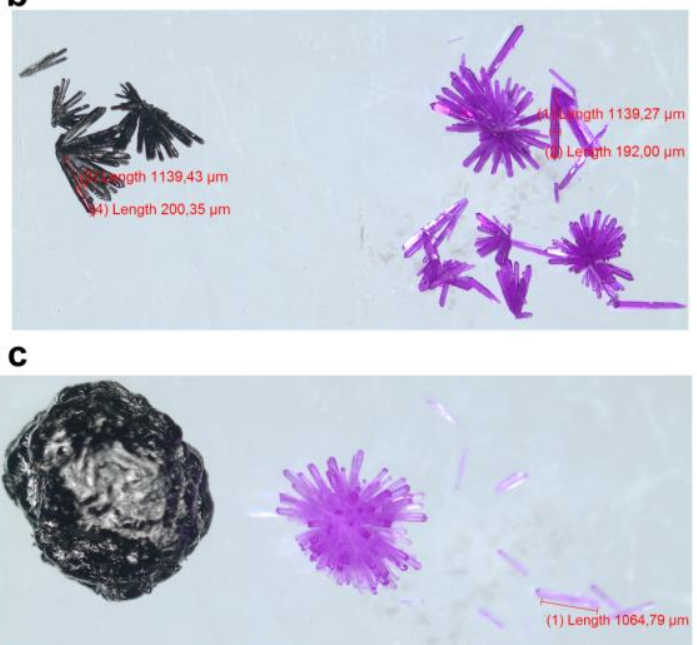

Fig. 4 | Thermal behavior of Co-doped Zn-ZIF-zni crystals. a, Phase diagram of $\mathrm{Co}_{\mathrm{x}} \mathrm{Zn}_{1-\mathrm{x}}-\mathrm{ZIF}-\mathrm{zni}$ ( $\mathrm{x}=0.3$ to 0.003 ) solid solutions. $T_{m}$ is the offset temperature of the calorimetric melting peak in DSC plots while $T_{d}$ is the extrapolated onset decomposition temperature on the TGA curves (Fig. S22) obtained from simultaneous TGS/DSC experiments. The respective $T_{m}$ and $T_{d}$ for the ultralow mole fractions of Co $(x=0.01$ to 0.003$)$ in Co $_{x} Z_{1-x}-Z I F-z n i$ is shown in inset. b-c, Optical images of two solid solution sample (b) $\mathrm{Co}_{0.04} \mathrm{Zn}_{0.96}$-ZIF-zni, (c) $\mathrm{Co}_{0.01} \mathrm{Zn}_{0.99}$-ZIF-zni before heating (right-purple crystals) and after heating (left-black product) experiments in argon atmosphere upto $750{ }^{\circ} \mathrm{C}$. The scale of individual images is $1 \mathrm{~mm}$. 